\title{
Incidência de transtornos Psíquicos na gravidez e Puerpério em mulheres De 18 a 35 anos no Hospital Beneficente Portuguesa de Belém do Pará / Pa , Brasil
}

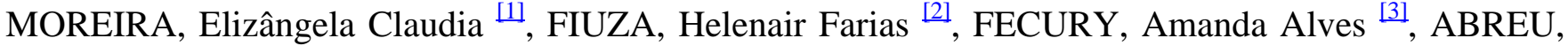 \\ Luciléa de Jesus Madeira Palhano de ${ }^{[4]}$, DIAS, Cláudio Alberto Gellis de Mattos ${ }^{[5]}$, OLIVEIRA, \\ Euzébio de ${ }^{[6]}$
}

MOREIRA, Elisângela Claudia. Et. al. Incidência de transtornos psíquicos na gravidez e puerpério em mulheres de 18 a 35 anos no Hospital Beneficente Portuguesa de Belém do Pará / PA, Brasil. Revista Científica Multidisciplinar Núcleo do Conhecimento. Ano 01, Ed. 05, Vol. 02, pp. 81-93, Maio de 2016. ISSN:2448-0959

\section{RESUMO}

De um modo geral as pessoas apresentam alterações no humor à medida que acontecimentos agradáveis ou desagradáveis vão surgindo em suas vidas. Na gravidez, ocorrem sérias transformações na mulher, tais como: modificação de seu corpo e de hormônios necessários para que aconteça a maturação do feto. Os transtornos psíquicos no puerpério estão entre os mais graves na psiquiatria. O presente estudo teve como objetivo determinar a incidência de Transtornos Psíquicos (Disforia, Depressão e Psicose) na gravidez e puerpério; em mulheres de 18 a 35 anos, primíparas e multíparas de diferentes classes sociais internadas no Hospital Beneficente Portuguesa de Belém, buscando identificar as possíveis causas que levem a esses transtornos na gravidez e no puerpério. Os transtornos psíquicos parecem ser muitas vezes subnotificados, sendo a Disforia a mais encontrada nesse estudo. A faixa etária de 18 a 24 anos com baixa renda mensal parecem ser fatores de risco associados aos distúrbios. O acompanhamento devido a essas gestantes durante o pré-natal poderia evitar estes tipos de transtornos.

Palavras-chave: Gravidez, Distúrbios Psíquicos, Saúde.

\section{INTRODUÇÃO}

De um modo geral as pessoas apresentam alterações no humor à medida que acontecimentos agradáveis ou desagradáveis vão surgindo em suas vidas. A intensidade e duração desses humores devem ser 
proporcionais aos momentos de vida e as condições psicoemocionais do sujeito. Na visão da psicanálise e da psicologia, isso é considerado normal. Dessa forma, os transtornos psicológicos podem ocorrer a todos os indivíduos independentemente de classe, cor, ou poder aquisitivo. A forma como estes transtornos se manifestam vai depender da vulnerabilidade deste indivíduo a eventos naturais e as predisposições psicossociais. Um dos momentos importante, e normalmente significativo, é a gravidez (CAMACHO et al., 2006).

$\mathrm{Na}$ gravidez, ocorrem sérias transformações na mulher, tais como: modificação de seu corpo e de hormônios necessários para que aconteça a maturação do feto. Essas modificações acabam gerando dúvidas, sentimento de fragilidade, insegurança e ansiedade (MOREIRA et al., 2008).

Durante a gestação e o puerpério a mulher vivencia grandes modificações, tanto físicas, como psíquicas e também em seu papel na família e na sociedade. Há alterações em sua auto-imagem corporal, conflitos relativos a feminilidades, à sexualidade e até mesmo à gravidez (ZANOTTI et al., 2003).

No estado puerperal a mulher passa por profundas alterações físicas, psicológicas e sociais. As alterações psicológicas podem ser traduzidas na ideia de que, na visão da mulher, a gravidez é a realização do sonho de ser mãe. Entretanto, devido ocorrer transformações significativas no corpo e modificações hormonais, sendo ou não planejada e desejada, poderá desencadear conflitos psicológicos como: disforia, depressão pós-parto e psicose puerperal, provocando muitas vezes, sentimento de fragilidade, insegurança e ansiedade. Principalmente, em casos de mulheres primíparas, onde a gravidez se torna uma novidade associada à falta de experiência, causando sentimentos de medo e temor de não saber como lidar com a situação (CAMACHO et al, 2006; MOREIRA et al., 2008).

Zanotti et al (2006), afirmaram que na fase puerperal a mulher encontra-se exposta a maiores riscos de aparecimentos de transtornos mentais em relação a outras fases da vida, uma vez que as duas defesas, tanto físicas, quanto psicossociais, são direcionadas a proteção e vulnerabilidade do bebê.

Segundo Neme (2005) e Moura et al., (2011), os transtornos psíquicos no puerpério estão entre os mais graves na psiquiatria. Apresentando risco tanto para mãe assim também como para o recém-nascido, e os classificam em quadros mais leves chamado de Disforia no puerpério e depressão puerperal e em quadros mais graves, chamados de transtornos psicóticos.

A Disforia do pós-parto ou também chamado "Puerperal blues", onde ocorre mudança repentina de humor podendo haver também sentimentos como tristeza, angústia, melancolia, pessimismo e sentimento de pena, considerado por alguns autores, como um fenômeno fisiológico comum no puerpério (NEME, 2005).

As mudanças repentinas de humor depressivo aparecem nos primeiros dias do pós-parto e tende a diminuir no décimo dia do puerpério. Geralmente são sintomas depressivos leves acompanhados por instabilidade emocional, irritação e tensão que não chegam a comprometer a relação da mãe com o recémnascido, não havendo necessidade de tratamento médico, mais se a persistência desse humor não desaparecer a partir do décimo primeiro dia, pode ser encarada como uma possível depressão maior, necessitando de avaliação e tratamento adequado (NEME, 2005; CAMACHO et al., 2006).

A depressão é caracterizada por um estado de tristeza, transtornos do sono, sentimentos de culpa, 
indecisão, desânimo, anorexia, inadequação para atender o recém-nascido. Habitualmente, a depressão pode significar tanto um estado afetivo quanto um sintoma, síndrome de uma ou várias doenças (RIBEIRO e ANDRADE, 2005).

De acordo com Sotto-Mayor e Piccinini (2005); Fonseca e Silva (2010) e Moura et al., (2011), a depressão pós-parto é um estado depressivo transitório que aparece na maioria das mulheres após o nascimento do bebê.

O quadro clínico da Depressão Pós-Parto é muito variável, podendo apresentar alterações físicas, cognitivas e comportamentais, é caracterizada por sintomas como tristeza, choro fácil, desalento, abatimento, labilidade, anorexia, algumas vezes acompanhada com náuseas, transtornos no sono, principalmente insônia e pesadelos, incluído também os próprios cuidados com o bebê, tais como os sentimentos de não amá-lo o suficiente, medo de prejudicá-lo, ansiedade com o sono e a alimentação da criança, sentimento de incapacidade para um cuidado adequado (ZANOTTI et al., 2003).

A depressão pós-parto é um transtorno psiquiátrico que causa grande sofrimento para a mãe e perturba a interação mãe-filho, esposa-marido e a relação familiar (ZANOTTI et al., 2003; FONSECA et al., 2010; MOURA et al., 2011).

Um dos fatores que podem contribuir para alto índice de depressão na gravidez e puerpério em mulheres na adolescência é a falta de maturidade afetiva de relacionamentos dessas pacientes, gravidez indesejada devido a relacionamentos não resolvidos, assim como ao fato de muitas terem que deixar sua liberdade da juventude ou até mesmo ter que abandonar os estudos em razão da gestação (SZIGETHY, 2001).

A psicose puerperal representa o quadro e menos frequente, sendo sua incidência encontrada entre $1 \%$ para cada nascimentos (CARVALHO, 2007). Alguns fatores de risco para psicose no puerpério podem estar relacionados á história de transtorno psiquiátrico prévio, eventos estressantes, conflitos conjugais, ser solteira ou divorciada, ou apresentar pouco suporte social (CAMACHO et al., 2006).

A psicose puerperal encontra-se entre os quadros mais graves na psiquiatria, representando o risco tanto para a mãe quanto para o bebê. Desenvolve-se entre o terceiro e o décimo quarto dia pós-parto, esta relacionada à primiparidade e mães solteiras. Isto ocorre quando há história pessoal ou familiar de doença mental, as alterações hormonais e estresses psicossociais (NEME, 2005).

De acordo com Nunes Filho e Bueno (2005), a psicose pós- parto não deve ser confundida com a tristeza pós-parto, ("Puerperal blues") devido ser uma condição normal que acomete em cerca de 50\% das mulheres.

É importante que o profissional de saúde consiga identificar as características dos transtornos psíquicos na gravidez durante o pré-natal e no puerpério, tendo inicio nas três primeiras semanas do puerpério com alta incidência e trazendo consequências para a mãe e para criança. $\mathrm{O}$ profissional deve ter cuidado e atenção adequado as puérperas para identificar e intervir mais precocemente no tratamento e no conhecimento da patologia, pois a psicose é o quadro depressivo que tem alta prevalência, e divergências entre autores sobre os fatores relacionados a essa patologia (HIGUTI, 2003).

Sendo a gestação um processo normal do desenvolvimento humano, com grandes transformações 
orgânicas, psíquico e sócio familiar. A assistência prestada deve ser integral proporcionando a mulher satisfação e bem-estar, visando o fortalecimento do vinculo entre mãe-bebê (FALCONE et al., 2005).

Algumas mães sentem vergonha de demonstrar para seus médicos e familiares os sintomas depressivos, não conseguindo cumprir suas novas responsabilidades, surgindo nelas, o sentimento de culpa por não vivenciar este momento de forma satisfatória, havendo a necessidade de o profissional identificar estes sintomas, para interagir e deixar a mãe mais segura para expor o seu problema (PEIXOTO, 2007; GOMES et al., 2010).

O profissional deve ter como objetivo compreender as emoções, sentimentos, fantasias, temores e alívios às ansiedades presentes a paciente, observando estados de confusão na parturiente entre o período da gravidez e após o nascimento do bebê. Os sentimentos e reflexões a respeito da puérpera interferem e dificultam as atividades enfrentadas no dia-a-dia do profissional. Sendo assim, a assistência deverá identificar na puérpera o seu repudio ao bebê, tristeza, afastamento, ausência, inapetência, descuido de autocuidado, insônia, preservando o sono e repouso regular da puérpera para que possa enfrentar suas atividades requeridas com o bebê (TRUCHARTE et al., 2010; VIEIRA et al., 2011).

$\mathrm{O}$ atendimento esclarece, apoia e diminui a ansiedade da parturiente, ou seja, proporciona benefício à mulher, facilitando a expressão de seus sentimentos diante das alterações da maternidade. $\mathrm{O}$ tratamento inclui antidepressivos, abordagem psicoterápica, orientação, apoio, visitas domiciliares até a completa remissão do quadro depressivo e ou psicótico (ZANOTTI et al., 2003).

Com base no exposto, o presente estudo teve como objetivo determinar a incidência de Transtornos Psíquicos (Disforia, Depressão e Psicose) na gravidez e Puerpério, em mulheres de 18 a 35 anos, primíparas e multíparas de diferentes classes sociais internadas no Hospital Beneficente Portuguesa de Belém, buscando identificar as possíveis causas que levem a esses transtornos na gravidez e no puerpério.

\section{METODOLOGIA}

Este estudo foi feito através da pesquisa quantitativa, realizado por pesquisa em banco de dados, buscando identificar a incidência dos três estados de transtornos psíquicos na gravidez e puerpério (Disforia, Depressão, Psicose), em 100 mulheres de 18 a 35 anos, primíparas e multíparas de diferentes classes sociais atendidas na maternidade do Hospital Beneficente Portuguesa de Belém do Pará, no período de Março à Abril de 2014. Foram excluídas mães menores de 18 anos e maiores de 35 anos. O programa Excel do Office da Microsoft Corporation foi utilizado na organização e tabulação dos dados e para análise estatísticas.

\section{RESULTADOS}

\section{DURANTE A GRAVIDEZ}

Com base nos resultados coletados no banco de dados, das 33 mulheres que apresentaram sinais e sintomas de Disforia durante a gravidez, a maioria possuiu renda entre 1 e 1/2 salários mínimos, o que representa $42,4 \%$ dessas mulheres. Vinte e sete apresentaram sinais e sintomas de Depressão representando 44,\% que também possui renda entre 1 e 1/2 salário mínimo (Tabela 1). 
Tabela 1: Percentual da renda mínima das mulheres com transtorno psíquicos na gravidez.

\begin{tabular}{|c|c|c|c|c|c|c|}
\hline \multirow{2}{*}{ Renda Familiar } & \multicolumn{2}{|c|}{ Disforia } & \multicolumn{2}{|c|}{ Depressão } & \multicolumn{2}{|c|}{ Psicose } \\
\hline & Quantidade & $\%$ & Quantidade & $\%$ & QT. & $\%$ \\
\hline $1 \mathrm{e}$ 1/2 salários mínimo & 14 & 42,4 & 12 & 44,4 & 0 & 0,0 \\
\hline 2 e $2 \frac{1}{2}$ salários mínimo & 8 & 24,2 & 11 & 40,7 & 0 & 0,0 \\
\hline $\begin{array}{l}3 \text { a } 4 \text { salários mínimos } \\
\text { Acima de } 4 \text { salários }\end{array}$ & 8 & 24,2 & 4 & 14,9 & 0 & 0,0 \\
\hline mínimos & 3 & 9,2 & 0 & 0,0 & 0 & 0,0 \\
\hline Total & 33 & 100,0 & 27 & 100,0 & 0 & 0,0 \\
\hline
\end{tabular}

Fonte: Hospital Beneficente Portuguesa, Belém PA, 2014.

Trinta e três mulheres apresentaram sinais e sintomas de Disforia. Verificou-se que a maioria (66,7\%) são primíparas, e 33,3\% são multíparas. Das primíparas, 17 mulheres (51,5\%), apresentaram idade entre 18 e 24 anos. Das multíparas, 6 mulheres (18.2\%), apresentaram idade entre 18 e 24 anos. No total as mulheres que apresentaram disforia na faixa etária de 18 a 24 anos entre primíparas e multíparas compreendem $69,7 \%$, e as de faixas etárias entre 25 a 35 anos entre primíparas e multíparas totalizam $30,3 \%$. Com relação às mulheres com Depressão, verificou-se que a maioria são multíparas. Das 27 mulheres que apresentaram sinais e sintomas de Depressão, 15 eram multíparas representando 55,6\%, e 12 primíparas, representando 44,4\%, com destaque também na faixa etária de 18 a 24 anos, que totaliza entre primíparas e multíparas $66,7 \%$, e as demais faixas etárias uma minoria de 33,\%. Não se verificou casos de mulheres com transtornos de Psicose durante a gravidez (Tabela 2).

Tabela 2: Percentual de mulheres com transtorno psíquico durante a gravidez, na faixa etária de 18 a 35 anos, primíparas e multíparas. 


\begin{tabular}{|c|c|c|c|c|c|c|}
\hline \multicolumn{7}{|c|}{ DISFORIA } \\
\hline \multirow{2}{*}{ Faixa etária } & \multicolumn{2}{|c|}{ Primípara } & \multicolumn{2}{|l|}{ Multípara } & \multirow{2}{*}{ Total } & \multirow{2}{*}{$\%$} \\
\hline & Quantidade & $\%$ & Quantidade & $\%$ & & \\
\hline 18 a 24 anos & 17 & 51,5 & 6 & 18,2 & 23 & 69,7 \\
\hline 25 a 29 anos & 2 & 6,1 & 1 & 3,0 & 3 & 9,1 \\
\hline 30 a 35 anos & 3 & 9,1 & 4 & 12,1 & 7 & 21,2 \\
\hline Total & 22 & 66,7 & 11 & 33,3 & 33 & 100,0 \\
\hline \multicolumn{7}{|c|}{ DEPRESSÃO } \\
\hline \multirow{2}{*}{ Faixa etária } & Primípara & & Multípar: & & \multirow{2}{*}{ Total } & \multirow{2}{*}{$\%$} \\
\hline & Quantidade & $\%$ & Quantidade & $\%$ & & \\
\hline 18 a 24 anos & 10 & 37,0 & 8 & 29,7 & 18 & 66,7 \\
\hline 25 a 29 anos & 1 & 3,7 & 2 & 7,4 & 3 & 11,1 \\
\hline 30 a 35 anos & 1 & 3,7 & 5 & 18,5 & 6 & 22,2 \\
\hline Total & 12 & 44,4 & 15 & 55,6 & 27 & 100,0 \\
\hline \multicolumn{7}{|c|}{ PSICOSE } \\
\hline \multirow{2}{*}{ Faixa etária } & Primípara & & Multípar: & & \multirow{2}{*}{ Total } & \multirow{2}{*}{$\%$} \\
\hline & Quantidade & $\%$ & Quantidade & $\%$ & & \\
\hline 18 a 24 anos & 0 & 0,0 & 0 & 0,0 & 0 & 0,0 \\
\hline 25 a 29 anos & 0 & 0,0 & 0 & 0,0 & 0 & 0,0 \\
\hline 30 a 35 anos & 0 & 0,0 & 0 & 0,0 & 0 & 0,0 \\
\hline Total & 0 & 0,0 & 0 & 0,0 & 0 & 0,0 \\
\hline
\end{tabular}

Fonte: Hospital Beneficente Portuguesa, Belém PA, 2014.

\section{DURANTE O PUERPÉRIO}

Verificou-se que no puerpério, das 100 mulheres, 4 apresentaram sinais de Disforia a maioria com renda entre 1 e 1/2 salários mínimos, (50\%). Já em relação à Depressão, verificou-se que mais de $65 \%$ apresentaram renda mínima abaixo de 3 salários mínimos, e as com sinais de psicose, somente um caso (100\%) com renda entre 1 e 1/2 salário mínimo (Tabela 3).

Tabela 3: Percentual da renda mínima das mulheres com transtornos psíquico. 


\begin{tabular}{lrrrrrr}
\hline \hline \multirow{2}{*}{ Renda Familiar } & \multicolumn{2}{c}{ Disforia } & \multicolumn{2}{c}{ Depressão } & \multicolumn{2}{c}{ Psicose } \\
\cline { 2 - 8 } & Quantidade & \multicolumn{1}{c}{$\%$} & Quantidade & $\%$ & Quantidade & $\%$ \\
\hline 1 e 1 e meio salários & 2 & 50,0 & 1 & 33,3 & 1 & 100,0 \\
2 e 2 e meio salários & 1 & 25,0 & 1 & 33,3 & 0 & 0,0 \\
3 a 4 salários & 1 & 25,0 & 1 & 33,3 & 0 & 0,0 \\
Acima de 4 salários & 0 & 0,0 & 0 & 0,0 & 0 & 0,0 \\
\hline Total & 4 & 100,0 & 3 & 100,0 & 1 & 100,0 \\
\hline \hline
\end{tabular}

Fonte: Hospital Beneficente Portuguesa, Belém PA, 2014.

Quatro mulheres apresentaram sinais e sintomas de Disforia. Observou-se que a maioria (75\%) são primíparas na faixa etária de 18 a 24 anos, enquanto que, 25\% são multíparas na faixa etária de 30 a 35 anos. Já em relação às mulheres com depressão, verificou-se que 66,7\% são primíparas na faixa etária de 18 a 24 anos e 33,3\% são multíparas na faixa etária de 30 a 35 anos, enquanto que as mulheres com psicose, somente $1(100 \%)$ caso de mulher multípara com idade na faixa etária entre 30 e 35 anos (Tabela 4).

Tabela 4: Percentual de mulheres com transtorno psíquico puerperais, na faixa etária de 18 a 35 anos, primíparas e multíparas. 


\section{DISFORIA}

\begin{tabular}{|c|c|c|c|c|c|c|}
\hline \multirow{2}{*}{ Faixa etária } & \multicolumn{2}{|l|}{ Primípara } & \multicolumn{2}{|c|}{ Multípara } & \multirow{2}{*}{ Total } & \multirow{2}{*}{$\%$} \\
\hline & Quantidade & $\%$ & Quantidade & $\%$ & & \\
\hline 18 a 24 anos & 3 & 75,0 & & 0,0 & 3 & 75,0 \\
\hline 25 a 29 anos & 0 & 0,0 & & 0,0 & 0 & 0,0 \\
\hline 30 a 35 anos & 0 & 0,0 & & 25,0 & 1 & 25,0 \\
\hline Total & 3 & 75,0 & & 25,0 & 4 & 100,0 \\
\hline \multicolumn{7}{|c|}{ DEPRESSÃO } \\
\hline \multirow{2}{*}{ Faixa etária } & Primípara & & Multíp: & & \multirow{2}{*}{ Total } & \multirow{2}{*}{$\%$} \\
\hline & Quantidade & $\%$ & Quantidade & $\%$ & & \\
\hline 18 a 24 anos & 2 & 66,7 & & 0,0 & 2 & 66,7 \\
\hline 25 a 29 anos & 0 & 0,0 & & 0,0 & 0 & 0,0 \\
\hline 30 a 35 anos & 0 & 0,0 & & 33,3 & 1 & 33,3 \\
\hline Total & 2 & 66,7 & & 33,3 & 3 & 100,0 \\
\hline \multicolumn{7}{|c|}{ PSICOSE } \\
\hline \multirow{2}{*}{ Faixa etária } & Primípara & & Multíp: & & \multirow{2}{*}{ Total } & \multirow{2}{*}{$\%$} \\
\hline & Quantidade & $\%$ & Quantidade & $\%$ & & \\
\hline 18 a 24 anos & 0 & 0,0 & & 0,0 & 0 & 0,0 \\
\hline 25 a 29 anos & 0 & 0,0 & & 0,0 & 0 & 0,0 \\
\hline 30 a 35 anos & 0 & 0,0 & & 100,0 & 1 & 100,0 \\
\hline Total & 0 & 0,0 & & 100,0 & 1 & 100,0 \\
\hline
\end{tabular}

Fonte: Hospital Beneficente Portuguesa, Belém PA, 2014.

A Tabela 5 mostra que das mulheres, $60 \%$ apresentaram algum tipo de transtorno psíquico durante a gravidez. Destas, $83 \%$ não procuram a ajuda de um profissional de saúde, enquanto que, apenas, $17 \%$ procuraram um profissional de saúde. Das mulheres que procuraram ajuda de um profissional de saúde, $40 \%$ falou com psicólogo, enquanto que 30\% falou com enfermeiro, $10 \%$ falou com ginecologista e $20 \%$ falou com obstetra.

Tabela 5: Percentual de mulheres que apresentaram ou não transtornos psíquicos durante a gravides e que comunicaram a alguma profissional de saúde. 


\begin{tabular}{|c|c|c|}
\hline \multicolumn{3}{|c|}{$\begin{array}{c}\text { Mulheres que apresentaram ou não durante a gravidez } \\
\text { transtornos psíquicos }\end{array}$} \\
\hline & Quantidade & $\%$ \\
\hline Sim & 60 & 60,0 \\
\hline Não & 40 & 40,0 \\
\hline Total & 100 & 100,0 \\
\hline \multicolumn{3}{|c|}{$\begin{array}{l}\text { Mulheres com transtornos psíquicos que durante } \\
\text { a gravidez falaram ou não com profissional de saúde }\end{array}$} \\
\hline & Quantidade & $\%$ \\
\hline $\operatorname{Sim}$ & 10 & 16,7 \\
\hline Não & 50 & 83,3 \\
\hline Total & 60 & 100,0 \\
\hline
\end{tabular}

\section{Profissionais de saúde que as mulheres com transtornos psíquicos falaram durante a gravidez}

Profissional Quantidade

Ginecologista

Obstetra

Enfermeira

Psicólogo

Total

\begin{tabular}{ccc} 
Quantidade & & $\%$ \\
\hline & 1 & 10,0 \\
2 & 20,0 \\
3 & 30,0 \\
& 4 & 40,0 \\
\hline & 10 & 100,0 \\
\hline
\end{tabular}

Fonte: Hospital Beneficente Portuguesa, Belém PA, 2014.

DISCUSSÃO

O presente estudo teve a Disforia como o distúrbio mais comum. O predomínio foi de cerca de $66,7 \%$ das grávidas e 75\% no pós-parto, de primíparas na faixa etária de 18 a 24 anos com renda mensal de 1 e $1 / 2$ salário mínimo. Segundo Moreira et al., 2008, a gravidez afeta o funcionamento hormonal da mulher causando transformações corporais necessárias para que aconteça a maturação do feto, essas modificações acabam gerando dúvidas, sentimento de fragilidade, insegurança e ansiedade, levando ao quadro de disforia. Caracterizando-se por: mudança repentina de humor, podendo haver também sentimentos como 
tristeza, angústia, melancolia, pessimismo e sentimento de pena. Apesar de a disforia ser considerada como um distúrbio depressivo leve não necessitando de tratamento médico, sua persistência pode ser encarado como uma possível depressão maior, necessitando de avaliação e tratamento adequado (NEME, 2005).

Sinais e sintomas de depressão durante a gravidez foram encontrados em 55,6\% de multíparas e no pósparto de $66,7 \%$, apresentando também as mesmas faixas etárias e renda mensal. Pressupõe-se que o alto índice nessa faixa etária pode estar relacionado à falta de experiência, imaturidade, a gravidez indesejada devido relacionamentos não resolvidos, assim como ao fato de muitas terem que deixar sua liberdade da juventude ou até mesmo ter que abandonar os estudos em razão da gestação (SZIGETHY; RUIZ, 2001).

Com relação à psicose puerperal, esta representa o quadro mais grave e menos frequente, sendo sua incidência encontrada entre $1 \%$ para cada nascimento (CARVALHO, 2007).

Neste estudo, das 100 mães apenas uma multípara na faixa etária de 30 a 35 anos apresentou sintomas de psicose tais como: insônia, inquietação, irritabilidade, pranto, dificuldade de concentração. Pressupõe-se que a gestação tardia, a multiparidade, história de transtorno psiquiátrico, conflitos conjugais, ser solteira ou divorciada, ou apresentar pouco suporte social, podem ser fatores de risco para este transtorno (CAMACHO et al., 2006).

Um fato que merece destaque neste estudo é que das 100 mulheres que apresentaram algum tipo de transtorno psíquico durante a gravidez apenas, 17\% procurou um profissional de saúde para relatar seus sintomas. Os 83\% restantes, ou seja, a maioria, não relatou os sintomas. Acredita-se que por receio de possível estigmatização (EPPERSON, 1999); por algumas mães sentirem vergonha de demonstrar para seus médicos os sintomas; por acharem que não conseguem cumprir suas novas responsabilidades, surgindo nelas, o sentimento de culpa por não vivenciar este momento de forma satisfatória, sentindo tristes, infelizes, num momento em que deveriam estar se sentindo alegres (PEIXOTO; AMORIM, 2007).

Acredita-se que o bom atendimento esclarece, apoia e diminui a ansiedade da parturiente, facilitando a expressão de seus sentimentos diante das alterações da maternidade (ZANOTTI et al, 2003).

\section{CONCLUSÃO}

Os transtornos psíquicos parecem estar mais frequentes na gravidez e não puerpério, sendo muitas vezes subnotificados. A Disforia o quadro de maior incidência, e apesar de ser considerada como um distúrbio depressivo leve, geralmente não necessitando de tratamento médico, sua persistência pode ser encarada como uma possível depressão maior, necessitando de avaliação e manejo terapêutico adequado. A faixa etária de 18 a 24 anos com baixa renda mensal parecem ser fatores de risco associados a esses distúrbios.

O acompanhamento devido às gestantes durante o pré-natal poderia evitar estes tipos de transtornos. Para isso, os profissionais precisam estar habilitados, dispostos a ouvir atentamente as gestantes e puérperas para identificar e intervir mais precocemente no tratamento e no conhecimento da patologia.

\section{REFERÊNCIAS}

CAMACHO, R.S. et al. Transtornos psiquiátricos na gestação e no puerpério: classificação. Diagnóstico e 
tratamento. Revista de Psiquiatria Clínica. Clin. Vol. 33, nº 2- São Paulo, 2006.

CARVALHO, Geraldo M. Enfermagem em Obstetrícia. 3 ed. rev. e ampl. São Paulo: E.P. U, 2007.

EPPERSON, CN. Postpartum major depression: detection and treatment. Am Fam Physician 59 (8): 2247-2254, 2259-2260, 1999.

FALCONE, V. M. et al. Atuação multiprofissional e a saúde mental de gestantes. Revista Saúde Pública, São Paulo, mar. 2005, v. 39(4): 612-8.

FONSECA, V. R. J. R. M.; SILVA, G. A. D.; OTTA, E. Relação entre depressão pós-parto e disponibilidade emocional materna. Cad. Saúde Pública, v. 26, n. 4, p. 738-746, 2010.

GOMES, L. A. et al. Identificação dos Fatores de Risco para Depressão Pós-Parto: Importância do Diagnóstico Precoce. Rev. Rene, v. 11, p. 117-123, 2010.

HIGUTI, P. C. L.; CAPOCCI, P. O. Depressão pós-parto. Revista de Enfermagem da UNISA, 2003; 4: 46-50.

MOREIRA, T. M. M et al. Conflitos vivenciados pelas adolescentes com a descoberta da gravidez. Rev. Esc. Enferm. USP, 2008: 32 (2) 312-20.

MOURA, E. C. C.; FERNANDES, M. A.; APOLINÁRIO, F. I. R. Percepção materna sobre transtornos psiquiátricos no puerpério: implicações na relação mãe-filho. Rev Bras Enferm, v. 64, n. 3, p. 445-50, 2011.

NEME, Bussâmara- 1975. Obstetrícia Básica. 3 ed. São Paulo, 2005.

NUNES FILHO, E. P.; BUENO, J. R.; NARDI, A. E. Psiquiatria e saúde mental: conceitos clínicos e terapêuticos fundamentais. São Paulo: Editora Atheneu, 2005.

PEIXOTO, D. F.; AMORIM, V. C. O. Da Psicoembriologia ao puerpério: sensibilização à relação mãebebê. Revista Eletrônica de Psicologia. Ano 1, n. 1, jul. 2007.

RIBEIRO, W. G.; ANDRADE, M. O papel do enfermeiro na prevenção da depressão pós-parto (DPP). Informe-se na prevenção da saúde, v.5, n.1. P. 07- 09, 2009.

SOTTO- MAYOR, I. M. B.; PICCININI, C. A. Relacionamento conjugal e depressão materna. Psico. v. 36, n. 2, p. 135-138, maio/ago., 2005.

SZIGETHY, E.M.; RUIZ, P. Depression among pregnant adolescents: an integrated treatment approach. Am J Psychiatry 158 (1): 22-27, 2001.

TRUCHARTE, F.A.R.; KNIJNIK, R. B.; SEBASTIANE. R. W.; ANGERAMI- CAMON, V.A (organizador). Psicologia Hospitalar: Teoria e prática 2 edição revista e ampliada. São Paulo: Cengage Learning, 2010. 
VIEIRA, A. P. et al. Prevalência de Depressão Pós-parto em Mulheres de um Hospital Universitário. Anuário da Produção de Iniciação Científica Discente, v. 14, n. 25, p. 311-321, 2011.

ZANOTTI, D. V et al. Identificação e intervenção os transtornos psiquiátricos associados ao puerpério: a colaboração do enfermeiro psiquiatra. Revista Nursing, v. 61, n. 6, p. 36-42, jun. 2003. 1 CD-ROM.

[1] Psicóloga. Mestre em Teoria e Pesquisa do Comportamento. Docente da Universidade do Estado do Pará - UEPA. Pesquisadora do Programa de Pós Graduação do Núcleo de Medicina Tropical da UFPA.

${ }^{[2]}$ Acadêmica de Enfermagem da Faculdade Metropolitana da Amazônia.

[3] Biomédica. Doutora em Doenças Tropicais. Pesquisadora do Programa de Pós Graduação do Núcleo de Medicina Tropical da UFP. Docente e Pesquisadora da Universidade Federal do Amapá, AP.

[4] Acadêmica de Enfermagem da Faculdade Metropolitana da Amazônia. Pesquisadora do Programa de Pós Graduação do Núcleo de Medicina Tropical da UFPA.

${ }^{[5]}$ Biólogo. Doutor em Teoria e Pesquisa do Comportamento. Docente e Pesquisador do Instituto Federal do Amapá - IFAP.

[6] Doutor em Medicina / Doenças Tropicais. Pesquisador do Programa de Pós Graduação do Núcleo de Medicina Tropical da UFPA. Docente e Pesquisador na Universidade Federal do Pará - UFPA.

\section{PUBLIQUE SEU ARTIGO CIENTÍFICO EM:}

https://www.nucleodoconhecimento.com.br/enviar-artigo-cientifico-para-submissao 